Ann. Génét. Sél. anim., I973, 5 (I), I43-I45.

NOTE

\title{
CONTRIBUTION A L'ÉTUDE DE L'ALBINISME EN RACE BRUNE DES ALPES $\left.{ }^{1}\right)\left({ }^{2}\right)$
}

\author{
G. SUCCI \\ Institut de Zootechnie générale de la Faculté d'Agriculture, \\ Université de Milan, Via Celoria 2, \\ 20133 Milan (Italie) \\ RÉSUMÉ
}

Depuis 1967, quatre cas d'albinisme ont été décelés en race Brune des Alpes d'Italie (I femelle, 3 mâles), dans la zone de deux centres d'insémination. Des croisements expérimentaux ont été faits à partir de deux d'entre eux ; ils tendent à prouver que le caractère est déterminé par un facteur mendélien autosomal récessif, comme pour les cas très semblables extérieurement observés en Suisse ces dernières années. On pense qu'il s'agit du même gène qui aurait migré de Suisse en Italie. Les études histologiques, histochimiques et biophysiques montrent que le pigment mélanique est absent de la peau bien que le système enzymatique qui permet sa production semble présent. Dans le poil, on décèle parfois une faible quantité de pigment en liaison avec une coloration atténuée. Des études italo-suisses sont mises en place pour vérifier l'hypothèse d'une étiologie génétique commune et pour préciser les aspects histologiques, histochimiques et biophysiques de la mutation dans la peau, le poil et le sperme.

Suite à la publication de l'article de WEBER et LAUVERGNe (1964) relatif à l'apparition de cas d'albinisme en race bovine Brune des Alpes de Suisse, une enquête a été entreprise en Italie, avec la collaboration de l'Association Nationale des Eleveurs de la Race Brune des Alpes, auprès des deux principaux centres d'insémination qui travaillent avec cette race : le centre de Rovereto (Trentin-Haut Adige) et celui de Reggio Emilia (Émilie-Romagne).

Au début de I 967 un premier cas était signalé tout près de Trente (un mâle, né le 27-I I-66). Peu après on apprenait l'existence de deux autres veaux albinos, tous les deux mâles, nés comme le premier fin I966, l'un à Castellano près de Rovereto en octobre et l'autre en décembre à Vervo, près de Trente. Les deux premiers nés avaient pour père Stelvio, le troisième Secondo Bruno deux taureaux du centre de Rovereto qui, à la suite de ces découvertes, ne furent plus utilisés.

Deux ans plus tard, en septembre I968, une femelle albinos voyait le jour près de Castelnuovo Monti dans la zone du centre de Reggio Emilia. Le père était un taureau du centre importé de Suisse (Orell, 16873, Luzern-Emmen).

(1) Recherche financée par le Consiglio Nazionale delle Ricerche (C. N. R.), Roma.

(3) Cette note reprend et complète une communication au $X^{\mathrm{e}}$ Congres de Zootechnie (Paris-Versailles, juillet 197I), communication qui ne sera pas éditée dans les comptes rendus de cette manifestation (Bonadonna et Succi, 197I). 
Dès 1967 un des mâles apparu en Trentin, Haut-Adige, était acquis par l'Institut de Zootechnie générale de la Faculté d'A griculture de Milan (nº d'expérience : A I45), en 1968, c'était le tour de la femelle (A 147).

Accouplés, ces deux albinos ont déjà donné deux descendants albinos mâles (A I 5 I et A I 55). Croisé à deux vaches de race frisonne pie noire, le mâle albinos a donné un veau mâle et un veau femelle présentant la couleur habituelle des veaux croisés Brun des Alpes $\times$ Frison.

Extérieurement les albinos nés en Italie et décrits par ZANOTTI et al. (I967) ressemblent beaucoup aux albinos bruns des Alpes de Suisse, étudiés par Winzenried et Lauvergne (I970): absence de pigmentation mélanique dans les téguments, les phanères et la rétine, l'iris présentant une coloration bleu-vert clair. Avec l'âge, le manteau peut plus ou moins brunir, et dans un cas au moins, (le mâle acheté par l'Institut de Milan), la couleur bleu-vert de l'iris n'est apparue qu'asse $z$ tardivement, à l'âge de 35 mois.

D'une manière générale ce type d'albinisme ne semble guère altérer les performances zootechniques des animaux qui le portent. Comme anomalies pouvant résulter de la pléiotropie du facteur, on a toutefois noté un cas de cécité (en Suisse) et deux variations du nombre des trayons (une fois 6 et une fois 2 trayons).

$\mathrm{Vu}$ les similitudes phénotypiques observées et les liaisons bien connues entre la brune des Alpes italienne et la brune des Alpes suisse, on peut avancer avec une grande vraisemblance qu'il s'agit du même facteur mendélien récessif autosomal dans les deux rameaux.

En Italie les recherches ont surtout porté sur l'histologie et l'histochimie de la peau et sur certaines caractéristiques biophysiques du poil.

Dans la peau, on a relevé la présence de dopa-oxydase avec la même intensité et la même distribution que chez des bovins normaux. Ceci indiquerait que l'appareil enzymatique pour la production de mélanine existe, bien qu'on ne puisse mettre en évidence histochimiquement aucune trace de mélanine dans la peau (Rizzotri et al., i969).

Dans le poil, la recherche de la mélanine a été faite au moyen de la technique du signal EPR (résonnance paramagnétique électronique) avec laquelle Blors et al. (I96I) ont montré que le paramagnétisme des mélanines naturelles et synthétiques était indépendant de la couleur. On peut ainsi déceler dans les poils albinos de la mélanine, même en faible dose, et, partant, apparemment absente. Chez deux veaux albinos, il n'y avait aucun signal EPR, donc pas de mélanine avec des électrons libres. Chez un troisième animal examiné très jeune, on décelait, par contre, un signal EPR, considérablement plus faible que celui observé normalement en race Frisonne ou Brune des Alpes, (Zanotri et al., 1967). Cependant les poils d'un des animaux qui, tout jeune, n'avait présenté aucun signal EPR, ont, par la suite, présenté cette caractéristique, à mesure d'ailleurs que se développait un certain brunissement du pelage comme on le remarque parfois (cf. plus haut) (Rizzortr et al., r969).

Chez le taureau A 145 la première récolte de sperme a pu être faite à l'âge de I 3 mois. Les caractéristiques analysées (temps de réaction, volume de l'éjaculat, densité, motilité et concentration en spermatozoïdes) étaient subnormales, ne permettaient pas alors la congélation (BoNADonna et al., I969). Depuis une amélioration a été notée, qui a autorisé la congélation du sperme dans l'azote liquide. L'animal a été ensuite abattu.

Les caractéristiques ci-dessus prises en considération se sont révélées normales chez le second mâle étudié en Italie (A I 5I). On a commencé, en outre, l'étude de l'activité mélanisante du sperme de cet animal. Depuis juillet 1972, un échange de sperme congelé d'albinos s'est établi avec la Suisse où un troupeau d'albinos est entretenu à l'Institut de Zootechnie de l'Université de Zurich, (WinzenRIED et LAUVERGNe, I973). Il s'agit de vérifier l'hypothèse d'identité des deux mutants. De même les études, histologiques, histochimiques et biophysiques seront étendues à du matériel provenant de Suisse. Les comparaisons des pedigrées sont, quant à elles, en cours de réalisation mécanographiquement en collaboration avec le Département de Génétique et de Sélection animale de l'I. N. R. A., Jouy en Josas, France. 


\section{SUMMARY}

CONTRIBUTION TO THE STUDY OF ALBINISM IN THE BROWN SWISS CATTLE

Four cases of albinism were found in the Italian brown swiss breed ( 3 males, I female) since 1967 in the zone of two insemination centers. Experimental crosses were made using two of them. They seem to prove that the character is determined by a Mendelian recessive autosomal factor, as in similar cases externally observed in Switzerland in the past few years. It seems to be the same gene which migrated from Switzerland to Italy. Histological, histochemical and biophysical studies show that the melanic pigment is absent in the skin although the enzymatic system permitting its production seems to be present. A small quantity of pigment may sometimes be detected in the hair in relation to a pale color. Italo-Swiss studies are being done to check the hypothesis of a common genetic etiology and to define more accurately the histological and histochemical aspects of the mutation in the skin, hair and sperm.

\section{RIASSUNTO}

\section{CONTRIBUTO AGLI STUDI DELL' ALBINISMO NELLA RAZZA BRUNA ALPINA}

Dal 1967 , in Italia, nella razza Bruna Alpina, sono stati segnalati quattro casi di albinismo ( 1 fęmmina e 3 maschi) nella zona di attività di due Centri di Fecondazione Artificiale. Utilizzando due di essi, si sono compiuti alcuni incroci tendenti a provare che il carattere è detern inato da un fattore mendeliano autosomico recessivo, come per i casi, molto simili fenotipicamente, osservati in Svizzera in questi ultimi anni. Si pensa che si tratti dello stesso gene che sarebbe migrato dalla Svizzera in Italia. Gli studi istologici, istochimici e biofisici hanno dimostrato che la melanina è assente nella cute anche se il corredo enzimatico, che permette la sua sintesi, sembra presente. Nei peli si è rilevata la presenza di melanina, anche se in quantità limitata. Sono iniziati studi in collaborazione con la Svizzera per verificare l'ipotesi di un determinismo genetico comune e per precisare gli aspetti istologici, istochimici e biofisici della mutazione nella c.te, nei peli e nel material spermatico.

\section{RÉFÉRENCES BIBLIOGRAPHIQUES}

Blois M. S., Brown H. W., Lemmon R. M., Lindblau R. O., Weisbluth M., ig6r. Freeradicals in biological systems. Academic Press, New York, London.

Bonadonna T., Succi G., I97r. Certaines caractéristiques somatiques et comportement sexuel de bovins albinos. $X^{\mathrm{e}}$ Congrès int. Zootechnie. Thème VII (non édité)

Banadonna T., Roy Choudhury P. N., Succr G., I969. Osservazioni su talune caratteristiche del materiale spermatico di un toro albino di razza bruna delle Alpi. Boll. Soc. ital. biol. sperim., 46 (2), 49-51.

Rizzotti M., Aureli G., Zanotti L., Straini Panelli R., Succi G., ig69. Ricerche morfologiche e istochimiche sulla pelle e biofisiche sui peli di bovini albini. Riv. istoch. norm. pat., 15, (I, 2), 9I-Iro.

Weber W., Lauvergne J.-J., I964. Trois cas d'albinisme rencontrés en Suisse dans la race Brune des Alpes. Ann. Zootech. 13, I5I-I54.

Winzenried H. U., Lauvergne J.-J., 1970. Spontanes Auftreten von Albinos in der Schweizerischen Braunviehrasse. Schroiz. Arch. Tierheilk., 112, 58r-587.

Winzenried H. U., LaUvergne J.-J., r973. Recherches sur les gènes à effets visibles chez les bovins Schweiz. Arch. Tierheilk., 115, 95-105.

Zanotti L., Aureli G., Rizzotti M., Succi G., r967. Osservazioni su peli di bovini albini mediante lo studio di spettri EPR. Riv. istoch. norm. pat., 13 (5), I-ıo. 